\title{
La aplicación del marketing en hospitales públicos desde la perspectiva de los funcionarios: El caso de un hospital del sur de Chile
}

\author{
Jorge Riveros $\mathbf{S}^{1 a}$, Carmen Berné $\mathbf{M}^{2 b}$.
}

\section{Application of marketing strategies for the management of public hospitals from the viewpoint of the staff members}

Background: The implementation of the marketing strategies in public hospitals provides management advantages and improves the relationship between customers and staff. Aim: To analyze the application of marketing strategies in a public hospital, from the perspective of the staff. Material and methods: A structured survey that asked about perceptions in 50 items about communication between personnel and customers/ users, customer satisfaction, participation in the development of new policies and incentives for efficiency was applied to a stratified sample of the staff. Factorial and regression analyses were performed to define the impact of marketing strategies on the degree of preoccupation and orientation of the organization towards the satisfaction of customer needs. Results: The survey was applied to 74 males and 122 females. The survey showed that the orientation of the hospital towards the satisfaction of its beneficiaries basically depends on the generation of an organizational culture oriented towards them and the implementation of adequate policies in staff management and quality of service. These basic aspects can be accompanied with practices associated to the new marketing approaches such as a market orientation, customer orientation and relational marketing. All these factors presented positive and significant relations. Conclusions: New marketing strategies should be applied, to achieve an efficient and customer oriented hospital management (Rev Méd Chile 2006; 134: 353-60).

(Key w ords: Delivery of healthcare; Hospitals, public; Marketing of Health Services)

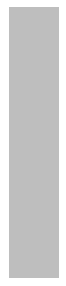

Recibido el 29 de marzo, 2005. Aceptado el 23 de diciembre, 2005.

Este trabajo de investigación cuenta con el patrocinio y financiamiento de la Dirección de Investigación de la Universidad Austral de Chile (Proyecto D.I.D. S 2003-68), no teniendo influencia en el diseño de estudio o en el tratamiento de los datos de investigación.

${ }^{1}$ Instituto de Administración, Universidad Austral de Chile. ${ }^{2}$ Departamento de Economía y Dirección de Empresas, Universidad de Zaragoza.

a Ingeniero Comercial, candidato a doctorado Universidad de Zaragoza (España).

${ }^{\mathrm{b}}$ Doctora en Ciencias Económicas y Empresariales.

Correspondencia a: Jorge Riveros S. Casilla de correo 567,

Valdivia, Chile. Fono-Fax: 63-221679. E mail: jriveros@uach.cl 
$\mathrm{L}$ os antecedentes disponibles, tanto teóricos como empíricos, llevan a plantear que en las instituciones del sector salud, las acciones se orientan hacia el número de prestaciones (orientación al producto) más que a la satisfacción del usuario. El marketing es una disciplina que tiene un amplio campo de aplicación en este sector ${ }^{1}$. Nuestro trabajo pretende señalar la importante contribución que el marketing puede aportar en la elección de la mejor orientación.

Dentro de este marco, este estudio tiene como objetivo identificar en una muestra representativa de funcionarios de un hospital del sur de Chile, los factores de aplicación del marketing y el peso relativo de cada uno, que permitirían una mayor orientación del hospital hacia la satisfacción de los usuarios.

En relación a la evolución del marketing y la utilidad de su aplicación en servicios públicos, se puede mencionar a Drucker ${ }^{2}$, quien fue uno de los primeros en establecer el concepto de marketing, al afirmar que la única definición válida para la meta de un negocio es crear clientes satisfechos. Toda actividad de gobierno puede ser considerada como "negocion, objeto de estudio del marketing ${ }^{3}$. Los programas, servicios y otros productos representan procesos de satisfacción de necesidades de la sociedad ${ }^{4}$.
Alderson y $\mathrm{Cox}^{5}$, proponen que si se pretende resolver los nuevos problemas de marketing planteados por los ámbitos públicos y privados, se requiere un nuevo paradigma: el del intercambio, propuesto inicialmente por Alderson ${ }^{6}$. Grönroos ${ }^{7}$ le entrega un aporte importante, al definir que "Marketing es establecer, mantener y aumentar las relaciones con los clientes y otros socios, para conseguir un beneficio. Esto se consigue mediante mutuo intercambio y cumplimiento de promesas". Este nuevo enfoque se conoce como Marketing de Relaciones.

A partir de la Economía Social de Mercado, se ha desarrollado una nueva filosofía empresarial llamada "Gestión dirigida al mercado" 8 , también conocida como Enfoque de Orientación al Mercado, que tiene un alcance más amplio que el marketing tradicional. Según Vázquez et al (1999), una empresa está orientada al mercado cuando cumple cuatro requisitos: "tener una clara orientación hacia el cliente y hacia la competencia, que esta orientación esté integrada al proceso de Dirección Estratégica; que disponga de herramientas, recursos, habilidades, capacidades y personal adecuados para desarrollar estrategias y políticas de marketing; y, que los resultados sean medibles en función de variables de mercado" (ver cita en Tabla 1).

\section{Tabla 1. Enfoque tradicional vs nuevos enfoques de marketing}

\begin{tabular}{|ll|}
\hline Enfoque Tradicional de Marketing & Nuevos Enfoques de Marketing \\
\hline Visión de corto plazo, desarrollo del & Visión de largo plazo, marketing como \\
marketing en un departamento. & filosofía organizacional. \\
Marketing masivo, escaso contacto, bajo & Marketing personalizado, contacto continuo \\
compromiso con cliente. & y alto compromiso. \\
Preocupación por los atributos del & Preocupación por proporcionar valor al \\
producto. Calidad endógena. & cliente. Calidad exógena. \\
Acciones dirigidas a atraer clientes y & Acciones dirigidas a atraer y retener clientes. \\
materializar ventas. & Gran énfasis en el servicio al cliente y en sus expectativas. \\
Poco énfasis en servicio al cliente. & Bases de datos, marketing interno y marketing interactivo. \\
Énfasis en el marketing mix: producto, & Considera al cliente como factor clave para \\
precio, distribución y comunicación. & la toma de decisiones. \\
& Dispone de todos los recursos, capacidades y \\
& habilidades para dar satisfacción a los clientes. \\
& Estrategia de coordinación para dar \\
& satisfacción al cliente. \\
& Estrategia de generación de información sobre el cliente. \\
& Canales de comunicación estratégicos. \\
\hline
\end{tabular}

Fuente: Vázquez R, Santos M, Díaz A, Alvarez L. Estrategias de Marketing: Desarrollo de investigaciones sobre orientación al mercado y marketing de relaciones. Universidad de Oviedo, 1999. Documento de trabajo 172. (Publicación local de circulación restringida). 
Aun cuando este enfoque se ha desarrollado mayoritariamente en el ámbito empresarial tradicional, se reconoce la conveniencia de extenderlo hacia ámbitos diferentes. Por ejemplo, Narver y Slater? sugieren réplicas en otros entornos; Kohli et al ${ }^{10}$ plantean aplicarlo a otras formas organizacionales atípicas, otros sectores industriales y otros países.

Estos planteamientos, proveen las condiciones necesarias para la introducción del marketing y de sus nuevos enfoques en el ámbito público con la consiguiente orientación al mercado de sus instituciones. En mercados públicos, ésta es una orientación hacia el cliente/usuario, puesto que la competencia es menos significativa. Esta orientación es denominada también, "orientación al servicio del público" ${ }^{11}$.

\section{MATERIAL Y MÉTODO}

El hospital, sobre el que se realiza la aplicación empírica, se encuentra en una comuna del sur de Chile. Su radio de acción se extiende principalmente hacia su región, pero en algunas especialidades, lo hace también, hacia otras regiones. Se trata de un hospital moderno, que presta atenciones de nivel secundario y terciario y que se encuentra catalogado de alta complejidad.

Para la realización de este trabajo, se diseñó y aplicó una encuesta estructurada, en forma personal, a una muestra representativa y estratificada de funcionarios del hospital. De este modo, la perspectiva del análisis se centra en ellos, actores imprescindibles para el desarrollo de objetivos ligados a la satisfacción de usuarios.
El cuestionario, además de requerir información sobre datos personales y profesionales del encuestado, solicitaba percepciones referentes a un conjunto de 50 ítems, presentados en sentencias afirmativas sobre la ocurrencia de determinados hechos, en una escala Likert de 1 a 7 , desde "no, en absoluto" hasta "siempre". Estas afirmaciones se formularon de manera que fueran comprendidas por todos los niveles del hospital y contenían, tanto aspectos que revelaban la aplicación del marketing tradicional como la de nuevos enfoques. Para el diseño de las afirmaciones, se consideraron aspectos señalados por Vázquez et al (1999), como diferenciadores de estas tendencias de marketing (Tabla 1).

Una vez decidido el diseño en conjunto con autoridades del hospital, y con posterioridad a la aplicación de una prueba piloto a cinco funcionarios, para verificar la comprensión del instrumento, se procedió a la selección de la muestra, definiéndose como población al total de funcionarios del mismo, esto es, 1.655 personas a la fecha de realización del estudio. La muestra se determinó por medio de muestreo estratificado según escalafón y con afijación proporcional, clasificándose la población en estratos de acuerdo a su escalafón, y obteniendo luego por separado, una muestra aleatoria simple de cada uno de ellos.

La aplicación de la fórmula estadística para una muestra aleatoria de tamaño "n" con población finita, entregó como resultado una muestra de 196 funcionarios, con un nivel de confianza de $95 \%$ y un error de estimación de 6,55\%. La distribución resultante se encuentra en la Tabla 2.

Para el tratamiento posterior de los datos (análisis descriptivo, análisis fiabilidad, análisis

Tabla 2. D istribución del número de funcionarios de la muestra por escalafón

\begin{tabular}{|lcc|}
\hline Escalafón & Pob. de funcionarios & № de funcionarios encuestados \\
\hline Directivos & 23 & 3 \\
Médicos & 283 & 33 \\
Profesionales & 251 & 29 \\
Técnicos & 676 & 80 \\
Administrativos & 164 & 20 \\
Auxiliares & 258 & 31 \\
Total Funcionarios & 1.655 & 196 \\
\hline
\end{tabular}

Fuente: Elaboración propia a partir de Memoria del hospital 
factorial y análisis regresión), se utilizó el paquete estadístico computacional SPSS, versión 10.0.

Se pretendió básicamente, extraer una simplificación de la información reflejada en las distintas variables mediante análisis factorial ${ }^{12}$, con el propósito de agrupar las variables en factores que representaran aspectos diferenciados de aplicación del marketing, tanto tradicionales como de nuevos enfoques, en el establecimiento hospitalario, midiendo el impacto que tenían éstos, a través del análisis de regresión ${ }^{13}$, en la orientación general del mismo hacia la satisfacción de las necesidades de los usuarios.

\section{Resultados}

La muestra fue constituida por 74 hombres $(37,5 \%)$ y 122 mujeres $(62,5 \%)$; donde $31,4 \%$ tenía más de 20 años de antigüedad en el servicio, 31\% tenía entre 10 y 20 años y 37,6\% tenía menos de 10 años de permanencia en el hospital.

Análisis factorial. El total final de ítems considerados para el análisis ascendió a 47, todos relativos a la percepción sobre distintos aspectos de aplicación del marketing en el hospital. Se excluyeron dos afirmaciones de apreciación general, destinadas a ser variables a explicar y que se utilizaron para el análisis de regresión posterior y una eliminada por inconsistente. El Análisis de Fiabilidad $^{14}$ sobre esta escala de medición arrojó un Alpha de Cronbach de 0,97; lo que indica una alta fiabilidad del instrumento utilizado. Una vez probada la idoneidad de la aplicación, se utilizó
Análisis Factorial con componentes principales y rotación Varimax. Se obtuvieron así, seis factores o componentes principales que explicaban más de 63\% de la varianza total (Tabla 3).

La composición de variables asociadas a estos factores se visualiza en la Tabla 4.

El Factor 1, que tiene un alto índice de fiabilidad y que explica el mayor porcentaje de la varianza total (sobre 20\%), está compuesto por políticas vinculadas a la "gestión de recursos humanos y de atención de usuarios". Variables de clima organizacional como políticas de diálogo entre estamentos, preocupación por relaciones interpersonales e instancias de apoyo al desempeño, presentan una alta correlación con el factor. Los otros ítems incluidos tienen que ver con la evaluación de la calidad de atención de los usuarios y su satisfacción, la distribución de sus datos entre los servicios, la atención de reclamos, etc.

El Factor 2, que también posee un alto índice de fiabilidad y que explicó $13,4 \%$ de la varianza, se podría considerar relacionado con una "cultura orientada hacia la valoración del usuario", reflejado en la incorporación de variables como el ofrecimiento de alternativas de solución al usuario, la preocupación por una solución integral de sus problemas, etc.

El siguiente factor que emerge, Factor $3(9,5 \%$ de la varianza), está relacionado con "políticas de modernización y gestión del reconocimiento". Los ítems que se incluyen, referidos a la modernización, se relacionan con mejoras en infraestructura. Los del segundo aspecto se relacionan con políticas de evaluación del desempeño, reconocimiento del hospital y del usuario al trabajo realizado por

Tabla 3. Análisis de la varianza total explicada

\begin{tabular}{|lccc|}
\hline Componentes principales & Varianza total & \% Varianza & \% Varianza acumulada \\
\hline Factor 1 & 9.447 & 20.100 & 20.100 \\
Factor 2 & 6.303 & 13.412 & 33.511 \\
Factor 3 & 4.477 & 9.526 & 43.037 \\
Factor 4 & 3.380 & 7.192 & 50.229 \\
Factor 5 & 3.150 & 6.702 & 56.931 \\
Factor 6 & 3.051 & 6.492 & 63.423 \\
\hline
\end{tabular}

Fuente: Elaborado en SPSS 10.0. Análisis de Componentes Principales y Normalización Varimax con Kaiser. 
Tabla 4. Composición de los factores o componentes principales

\begin{tabular}{|c|c|c|c|c|c|c|}
\hline Análisis factorial & $\begin{array}{c}\text { Factor } \\
1\end{array}$ & $\begin{array}{l}\text { Factor } \\
2\end{array}$ & $\begin{array}{l}\text { Factor } \\
3\end{array}$ & $\begin{array}{c}\text { Factor } \\
4\end{array}$ & $\begin{array}{l}\text { Factor } \\
5\end{array}$ & $\begin{array}{l}\text { Factor } \\
6\end{array}$ \\
\hline X40 existencia de políticas de diálogo entre estamentos & 0,737 & & & & & \\
\hline X38 preocupación por calidad de relaciones interpersonales & 0,728 & & & & & \\
\hline $\begin{array}{l}\text { X37 coordinación entre áreas o unidades } \\
\text { para realizar cambios internos }\end{array}$ & 0,712 & & & & & \\
\hline X41 instancias de apoyo al desempeño de los funcionarios & 0,710 & & & & & \\
\hline $\begin{array}{l}\text { X34 distribución de datos entre unidades } \\
\text { sobre satisfacción de usuarios }\end{array}$ & 0,691 & & & & & \\
\hline $\begin{array}{l}\text { X18 evaluación de unidades sobre calidad } \\
\text { v satisfacción de usuarios }\end{array}$ & 0,686 & & & & & \\
\hline X35 se recibe información de otras & 0,661 & & & & & \\
\hline $\begin{array}{l}\text { áreas sobre necesidades de usuarios } \\
\text { X43 participación en el diseño }\end{array}$ & 0,649 & & & & & \\
\hline modernizador del establecimiento & & & & & & \\
\hline $\begin{array}{l}\text { X19 medios comunicación internos con usuarios } \\
\text { (buzones de sugerencias,mesones de atención, etc.) } \\
\text { para sondear la calidad del servicio recibido }\end{array}$ & 0,629 & & & & & \\
\hline $\begin{array}{l}\text { X22 orientación masiva hacia buen uso de servicios } \\
\text { (folletos informativos) }\end{array}$ & 0,627 & & & & & \\
\hline X12 capacitación de funcionarios & 0,607 & & & & & \\
\hline $\begin{array}{l}\text { X39 creación de una identificación del personal } \\
\text { con respecto a obietivos del Centro Hospitalario }\end{array}$ & 0,592 & & & & & \\
\hline $\begin{array}{l}\text { X36 consideración necesidades usuarios en los } \\
\text { cambios a realizar }\end{array}$ & 0,563 & & & & & \\
\hline X17 consideración reclamos de los usuarios & 0,556 & & & & & \\
\hline X48 politicas de capacitación y desarrollo del personal & 0,551 & & & & & \\
\hline $\begin{array}{l}\text { X5 } \\
\text { X4 ofrecen alternativas solución al usuario } \\
\text { preocupación por dar solución integral }\end{array}$ & & $\begin{array}{l}0,749 \\
0,720\end{array}$ & & & & \\
\hline $\begin{array}{l}\text { X7 comunicación externa masiva por medios tradicionales } \\
\text { (prensa, radio, TV) destinada a entregar información }\end{array}$ & & 0,696 & & & & \\
\hline $\begin{array}{l}\text { X9 valoración del usuario en la planificación } \\
\text { estratégica de la atención }\end{array}$ & & 0,691 & & & & \\
\hline $\begin{array}{l}\text { X8 comunicación externa personalizada por medios } \\
\text { modenos (teléfono, e-mail, etc.) destinada a entregar } \\
\text { información oportuna }\end{array}$ & & 0,602 & & & & \\
\hline X33 reconocer cambios necesidades de usuarios & & 0,578 & & & & \\
\hline X11 incentivos a la calidad del trabajo & & 0,530 & & & & \\
\hline X49 políticas de evaluación del desempeño & & & 0,685 & & & \\
\hline $\begin{array}{l}\text { X46 hospital reconoce trabajo realizado } \\
\text { X14 mejoras en infraestructura }\end{array}$ & & & $\begin{array}{l}0,630 \\
0,608\end{array}$ & & & \\
\hline X45 modernización mejora clima interno de relaciones humanas & & & 0,602 & & & \\
\hline X44 modernización mejora atención usuarios & & & 0,562 & & & \\
\hline $\begin{array}{l}\text { X47 usuario reconoce trabajo realizado } \\
\text { X13 señalizán }\end{array}$ & & & 0,538 & & & \\
\hline $\begin{array}{l}\text { X13 señalización suficiente y adecuada } \\
\text { X15 preocupación por conocimiento de expectativas de usuarios }\end{array}$ & & & 0,504 & & & \\
\hline X26 toma de decisiones de áreas considera orientación & & & & 0,743 & & \\
\hline $\begin{array}{l}\text { X29 existencia de habilidades personal para aplicar } \\
\text { enfogue orientación al usuario }\end{array}$ & & & & 0,666 & & \\
\hline $\begin{array}{l}\text { X28 existencia de recursos materiales para aplicar } \\
\text { enfogue orientación al usuario }\end{array}$ & & & & 0,608 & & \\
\hline $\begin{array}{l}\text { X10 existencia de compromiso de estamentos por } \\
\text { satisfacer al usuario }\end{array}$ & & & & & 0,785 & \\
\hline X3 existe preocupación de funcionarios por satisfacer al usuario & & & & & 0,663 & \\
\hline $\begin{array}{l}\text { X32 conocimiento sobre la percepción de los usuarios } \\
\text { con respecto a la calidad del servicio }\end{array}$ & & & & & 0,555 & \\
\hline X25 existe utilización de base de datos & & & & & & 0,790 \\
\hline $\begin{array}{l}\text { X23 existe seguimiento individual post-atención } \\
\text { X24 existencia de base de datos actualizada }\end{array}$ & & & & & & 0,586 \\
\hline Alpha de Cronbach de cada Factor & 0,9486 & 0,8916 & 0,8710 & 0,7466 & 0,7177 & 0,7763 \\
\hline
\end{tabular}

Fuente: Elaborado en SPSS 10.0. Análisis de Componentes Principales y Normalización Varimax con Káiser. 
los funcionarios, así como la preocupación de éstos por conocer sus expectativas.

Los siguientes factores explican porcentajes más bajos de la varianza total. El Factor 4 está referido específicamente a la aplicación de prácticas del Enfoque de Orientación al Mercado o "prácticas de orientación hacia el usuario", ya que incluye ítems relativos a la consideración de éste en la toma de decisiones de las distintas áreas del hospital, así como contar con los recursos materiales y humanos necesarios para la implementación de este enfoque.

El Factor 5, denominado "compromiso de los funcionarios", incluye la preocupación por satisfacer las necesidades de los usuarios y el interés por conocer las opiniones de éstos, sobre la calidad del servicio. El sexto y último factor que emerge está relacionado con la aplicación del marketing desde una perspectiva de Marketing de Relaciones. Se incluyen en este factor tres variables que contemplan el seguimiento individual post-atención y la existencia y utilización de bases de datos actualizadas sobre usuarios. Denominamos a este último factor "gestión de relación con el usuario".

Podemos concluir por tanto, que en el conjunto de factores, se pueden observar algunos aspectos asociados a la aplicación del marketing tradicional, como también otros, asociados a la aplicación de las nuevas tendencias; y también a su vez, algunos factores que combinan ambos enfoques.

Análisis de regresión. Para llevar a cabo esta medición, seleccionamos dos variables de carácter general del cuestionario como variables dependientes, las que como señalábamos fueron excluidas del análisis anterior. La primera de ellas, mide el nivel de preocupación que tiene el hospital por satisfacer las necesidades de los usuarios, lo que podría traducirse como la misión del establecimiento. La segunda variable mide el grado de orientación que tienen los objetivos, planes y programas de funcionamiento para la satisfacción de los usuarios, lo que podría traducirse como la percepción que tienen los funcionarios sobre el funcionamiento integral del hospital destinado a la satisfacción de los usuarios. Ambas variables se encuentran bastante relacionadas y para efectos de esta presentación, consideraremos sólo la segunda, ya que logra un mayor nivel de ajuste para este tipo de análisis, aunque de todos modos es importante mencionar que, para ambos casos, los factores o componentes seleccionados por el análisis de regresión fueron los mismos y en un mismo orden de prioridad.

Por tanto, para el análisis de regresión (utilizando método stepwise), se consideró como dependiente la segunda variable indicada y como variables explicativas, los factores previamente definidos.

Los resultados obtenidos, mostrados en la Tabla 5, indican que de los seis factores analizados, cuatro de ellos, ejercían un efecto positivo y estadísticamente significativo ( $p<0,01)$. Los coeficientes estimados muestran que el Factor 2, relacionado con la aplicación del marketing en referencia a una cultura orientada al usuario, fue el que

\section{Tabla 5. Análisis de regresión sobre el funcionamiento del hospital para la satisfacción de sus usuarios}

\begin{tabular}{|lcrc|}
\hline $\begin{array}{l}\text { Variable dependiente: Orientación de objetivos, planes } \\
\text { y programas de funcionamiento del hospital para la } \\
\text { satisfacción de sus usuarios }\end{array}$ & $\begin{array}{c}\text { Coeficiente } \\
\text { Beta } \\
\text { estandarizado }\end{array}$ & $\begin{array}{c}\mathrm{t} \\
\text { value }\end{array}$ & Significatividad \\
\hline Constante & & 43,728 & 0,000 \\
Factor 2: Cultura orientada hacia la valoración del usuario & 0,517 & 6,974 & 0,000 \\
Factor 1: Gestión de recursos humanos y atención de usuarios & 0,323 & 4,363 & 0,000 \\
Factor 4: Orientación hacia el usuario & 0,316 & 4,265 & 0,000 \\
Factor 6: Relación con el usuario & 0,270 & 3,651 & 0,000 \\
$\mathrm{R}^{2}: 0,544 ; \mathrm{R}^{2}$ ajustado: 0,522 & & & \\
\hline
\end{tabular}

Fuente: Elaboración propia a partir de los resultados obtenidos en SPSS v.10.0. Análisis de regresión con método Stepwise. 
ejerció una mayor influencia sobre el funcionamiento del hospital para satisfacer las necesidades de sus usuarios. En segundo lugar se situaría el Factor 1, que incluye aspectos relacionados con la aplicación de esta disciplina en las políticas de recursos humanos y de atención al usuario. En tercer y cuarto lugar, influirían más específicamente las nuevas tendencias, tanto desde una perspectiva de orientación al usuario como desde una de relación con el usuario. Todas estas variables fueron capaces de explicar en más de 52\%, el impacto que tiene la aplicación del marketing sobre estos aspectos de funcionamiento del hospital, para la satisfacción de las necesidades de los usuarios, ya que el coeficiente de determinación, $\mathrm{r}^{2}$ ajustado, alcanzó un valor de 0,522.

\section{DISCUSIÓN}

Primero, hemos logrado el objetivo en términos de establecer cuáles son los factores más relevantes de aplicación del marketing y de sus enfoques más recientes, en la gestión de un establecimiento hospitalario público, desde la perspectiva de sus funcionarios; utilizando para ello una metodología de investigación cuantitativa y generando un instrumento de medición fiable que ha permitido aplicar herramientas estadísticas como análisis factorial y análisis de regresión.

Segundo, hemos confirmado que toda actividad de gobierno, y particularmente de servicio público, puede considerarse objeto de estudio y de aplicación del marketing.

Tercero, los factores extraídos reflejan la utilización tanto del marketing tradicional como de los nuevos enfoques, como son la Orientación al Mercado o al Usuario y el Marketing de Relaciones. Los enfoques por tanto, no son excluyentes entre sí y pueden concurrir perfectamente con el propósito de satisfacer apropiadamente las necesidades de los usuarios. Esta unión de enfoques se confirma con las expresiones de algunos expertos en el área que han señalado recientemente que hay que cerrar la actual separación que existe entre el marketing tradicional y el marketing relacional, manifestando además, que la Asociación Americana de Marketing (AMA) ha incluido en su última definición de la disciplina, la gestión de relaciones y la administración de procesos encaminados a crear más valor para el cliente ${ }^{15}$.

Cuarto, mediante el análisis de regresión, se ha obtenido el impacto que tiene la aplicación de estos factores de marketing en el nivel de preocupación del hospital por satisfacer las necesidades de sus usuarios y más específicamente en la orientación de sus objetivos, planes y programas de funcionamiento destinados a los mismos. Para ambos casos, la aplicación del marketing en lo que se refiere a la conformación de una cultura organizacional orientada al usuario es lo primordial. Le siguen, en un mismo orden y para ambos casos, la aplicación del marketing en las políticas de gestión de recursos humanos -que incluyen el manejo del clima organizacional- y en la calidad de atención al usuario. Luego, en tercer y cuarto lugar de incidencia, y también para ambos casos, la aplicación del marketing desde la perspectiva del Enfoque de Orientación al Mercado y la aplicación desde una perspectiva de Marketing de Relaciones; todos factores importantes a considerar en el logro de aumentar la satisfacción de los usuarios de estos centros de salud, desde la visión de sus recursos humanos.

Quinto, la consideración de los nuevos enfoques de marketing, como factores importantes en el logro de satisfacer las necesidades de los usuarios, incorpora al ámbito público la necesidad de un mayor y mutuo intercambio entre funcionarios y usuarios, lo que ayudaría a cambiar la mayor orientación al producto, que inicialmente comentábamos, por una mayor orientación al cliente o usuario. Estos aspectos de aplicación del marketing deberían integrarse efectivamente a los procesos de dirección de estos establecimientos hospitalarios.

Por último, como propósito futuro de nuestra investigación, debiéramos aplicar una encuesta similar a los usuarios para apreciar su punto de vista con respecto a la aplicación del marketing en los hospitales y si es que efectivamente puede contribuir a mejorar la satisfacción de sus necesidades de salud. Posteriormente se podrá realizar un estudio comparativo entre ambas visiones, lo cual será de sumo interés para la mejora de la gestión en los hospitales públicos. 


\section{REFERENCIAS}

1. Vázquez J, Placer J. Cinco temas de introducción al Marketing Público. León (España): Imprenta Moderna, 2000.

2. Drucker P. The Practice of Management. New York: Harper \& Row, 1954

3. ENIS B. Governments as marketers: Issues of management and public policy. En: Mokwa M y Permut S, eds. Government Marketing, Theory and Practice. Eds. Praeger, 1981; 343-56.

4. Cervera A. Marketing y Orientación al Mercado de la Administración Pública Local. Institució Alfons el Magnànim, Diputació de Valencia, 1999.

5. Alderson W, Cox R. Towards a Theory of Marketing. Journal of Marketing 1948; 13: 137-52.

6. Alderson W. Dynamic Marketing Behavior: a functionalist theory of marketing. Illinois: Richard D. Irwin, 1965.

7. GrönRoos C. Service Management an Marketing: Managing the «Moments of Truth» in Service Competition. Lexington Books y Macmillan Inc, 1990.

8. Lambin J. Ideas erróneas acerca del Marketing. En: Factores de Competitividad en el Comercio Mino- rista, Col-lecció estudis comercials, Generalitat Valenciana, 1999.

9. Narver J, Stater S. The effect of a Market Orientation on Business Profitability. Journal of Marketing 1990; 54: 20-35.

10. Kohli A, Jaworski B, Kuhmar A. Markor: A measure of Market Orientation. Journal of Marketing Research 1993; vol. XXX (4): 467-77.

11. Brugué Q, Amorós M, Goma R. La Administración Pública y sus clientes: ¿Moda organizativa u opción ideológica? Gestión y Análisis de Políticas Públicas 1994; 1: 34-46.

12. Hair J, Anderson R, Tatham R, Black W. Análisis Multivariante, $5^{\underline{a}}$ ed. Prentice Hall Iberia, 1999.

13. Peña D. Estadística: Modelos y Métodos 2. Modelos lineales y Series Temporales, $2^{\underline{a}}$ ed. Madrid: Alianza Universidad Textos, 1989.

14. Hayes B. Cómo medir la satisfacción del cliente. Oxford University Press México, 1999.

15. Alfaro M (Coordinador). Temas Clave en Marketing Relacional. McGraw Hill Interamericana de España, 2004. [Expresiones realizadas en la presentación de este libro en febrero de 2005 en Barcelona y disponibles en http://www. marketing-relacional.com, consultadas el 16 de noviembre de 2005].

\section{Agradecimientos}

Reconocemos la labor desarrollada por los académicos de la Universidad Austral de Chile, miembros del equipo de investigación del Proyecto D.I.D., Sres. Juan Carlos Miranda C. (Estadístico), Angélica Wenzel K. (Administrador Público), Enrique López R. (Administrador Público), Juan Carlos Bertoglio C. (Médico Cirujano) y María Cristina Torres (Enfermera y Matrona) quienes participaron en el diseño de la muestra y de la encuesta, en el análisis estadístico de la información, en asesoría técnica y administrativa y en distintos aportes a la realización del trabajo. 